\title{
Archéopages
}

Archéopages

Archéologie et société

Hors-série 3 | 2012

Nouveaux champs de la recherche archéologique

\section{L'organisation de l'archéologie préventive en Espagne et ses effets induits sur la recherche}

\section{Lauro Olmo Enciso}

Traducteur : Carine Carpentier

\section{OpenEdition Journals}

Édition électronique

URL : https://journals.openedition.org/archeopages/459

DOI : $10.4000 /$ archeopages.459

ISSN : 2269-9872

Éditeur

INRAP - Institut national de recherches archéologiques préventives

Édition imprimée

Date de publication : 1 janvier 2012

Pagination : $42-46$

ISSN : 1622-8545

Référence électronique

Lauro Olmo Enciso, "L'organisation de l'archéologie préventive en Espagne et ses effets induits sur la recherche », Archéopages [En ligne], Hors-série 3 | 2012, mis en ligne le 01 janvier 2012, consulté le 27 février 2023. URL : http://journals.openedition.org/archeopages/459; DOI : https://doi.org/10.4000/ archeopages. 459 


\section{L'organisation de l'archéologie préventive en Espagne et ses effets induits sur la recherche}

\author{
Lauro Olmo Enciso ${ }^{1}$ \\ Université d'Alcalá, Madrid
}

$\mathrm{L}$ es différences entre la France et l'Espagne en matière d'organisation de l'archéologie préventive se concentrent autour de deux aspects fondamentaux. Face à une structure centralisée en France, le modèle espagnol est défini par la décentralisation. Par ailleurs, face à une activité publique caractéristique du modèle français, l'intervention archéologique préventive en Espagne, contrôlée aussi par l'administration publique, est dans son exécution majoritairement entre les mains de l'initiative privée.

Le cadre légal et administratif. La mise en place de l'archéologie espagnole dans sa structure actuelle, est à mettre en relation avec la promulgation de la constitution de 1978 , fruit de réformes démocratiques et ayant pour objet la réorganisation de l'État. À partir de cette date commence la décentralisation politique et administrative qui aura pour conséquence la consolidation de l' «État des Autonomies », c'est-à-dire l'extension de l'autonomie politique régionale. De même, la rénovation épistémologique et méthodologique de la recherche archéologique espagnole durant les années 1980, similairement à ce qui se passe dans le reste de l'Occident, a conduit à parler, à partir de la seconde moitié de cette décennie, d'une nouvelle situation de l'archéologie. Cette décentralisation et cette rénovation, intimement liées au nouveau cadre légal (central, autonome et local), ainsi qu'aux prémices d'un processus de fort développement économique, laissaient envisager un important essor de lactivité archéologique en Espagne, tant d'un point de vue quantitatif que qualitatif.

La constitution espagnole de 1978 a profondément renouvelé l'ordre juridique sur le patrimoine historique. En matière d'archéologie les contributions émanant de la constitution supposent l'insertion de la protection des biens archéologiques dans les devoirs de l'État et, en outre, que cette protection reste décentralisée en faveur des 17 communautés autonomes. Un autre aspect intéressant est qu'elle fixe la prédominance des intérêts généraux sur les intérêts particuliers (art. 128.1) en établissant que tous les biens archéologiques appartiennent $\mathrm{au}$ « domaine public » et que, par conséquent, ils sont protégés par les principes d'inaliénabilité, d'imprescriptibilité et d'insaisissabilité (art. 132.1). Les biens archéologiques sont soumis à la protection l'État, mais celui-ci en assure aussi la promotion dans la ligne d'intervention propre de l' «État du Bien-être» (García Fernández, 2006, p. 535-536).

En Espagne, en conséquence de la structure décentralisée de l'État, toutes les compétences sur le patrimoine historique et sur l'urbanisme sont de la responsabilité directe des communautés autonomes. La constitution espagnole (art. 148) accorde ces compétences à ces communautés, tout en déclarant de la compétence exclusive de l'administration générale de l'État (art. 149, 1-28) la défense du patrimoine historique contre l'exportation et la spoliation.

L'Espagne s'est dotée d'une nouvelle «Loi du Patrimoine Historique Espagnol » (Loi 16/1985) approuvée par le parlement en 1985 (García Fernández, 2006, p. 37-39). Il s'agit d'une loi organique et, par conséquent, s'appliquant obligatoirement sur l'ensemble du territoire national et ne pouvant pas être modifiée par d'autres lois approuvées par les parlements des communautés autonomes. Les lois du patrimoine autonome, qui se sont succédé à partir de la décennie des années 1990, ont complété ou développé des aspects normatifs ou sectoriels, tels que ceux relatifs aux modèles de gestion. La loi étatique a introduit beaucoup de nouveautés, mais, en ce qui concerne les aspects qui sont ici traités, il faut souligner qu'elle a incorporé dans ses articles les formes prévues dans la législation urbaine, qui ont été développées postérieurement et, dans plusieurs cas, améliorées par les législations autonomes. En particulier a été introduit le caractère obligatoire d'effectuer des Cartes Archéologiques, instrument légal qui articulait une politique de prévention pour le patrimoine archéologique. C'est ainsi qu'est apparue une catégorie de zones définies comme «Ensemble Historique» ou «Zone Archéologique», pour lesquelles la loi considère l'obligation de rédiger des plans spéciaux qui s'occupent du patrimoine archéologique, et qui doivent contenir tous les aspects relatifs à la planification et à la protection de ces zones (Olmo Enciso, 2002, p. 246).

Suite à la nouvelle articulation autonome espagnole et le transfert des compétences en matière de patrimoine historique, par l'administration centrale de l'État aux administrations autonomes, ces dernières se sont dotées de leurs propres départements avec une responsabilité en matière archéologique. En ce sens, il convient de rappeler que l'ordre juridique autour du patrimoine archéologique se base sur le principe de domaine public (art. 44), ce qui suppose obligatoirement l'autorisation de l'administration pour toute intervention archéologique, son suivi et la décision finale du devenir des restes exhumés. Ont également été créés ou renouvelés les services archéologiques des administrations locales (mairies, conseils généraux). Une série de compétences sur le patrimoine ont été attribuées aux communes. En effet, la loi de patrimoine historique espagnol accorde aux communes une série d'obligations en vue de l'exécution de la loi et de leur coopération en matière de conservation et de surveillance du patrimoine historique. Elle souligne l'obligation de rédiger des «Plans Spéciaux de Protection pour les Biens d'Intérêt Culturel» (ensemble historique, site historique, zone archéologique), soit une forme de protection légale maximale devant être approuvée en amont par l'administration autonome. De même, la «Loi Régulatrice des Bases de Régime Local», établit (art. 25.2.e) que la commune aura des compétences sur le patrimoine historique dans les termes établis par la législation de l'État et des communautés autonomes, des compétences dérivées étant régulées par la mairie elle-même (Querol, Martínez, 1996, p. 195).
1 Traduction Carine Carpentier, Inrap. 
Cette décentralisation administrative a supposé une avancée indubitable et remarquable dans la recherche, la protection, la gestion et la promotion $\mathrm{du}$ patrimoine archéologique ces 25 dernières années, avec des dotations budgétaires et nombre d'investigations jusqu'alors impensables, tout ce qui permet de comprendre l'éclosion de la recherche archéologique en Espagne. Toutefois, d'un point de vue réglementaire, elle a aussi produit une hétérogénéité de situations, rendue propice par les divers niveaux de développement dans les lois et les règlements, ainsi que par les différents compromis administratifs, et surtout politiques, en ce qui concerne les stratégies de prévention et de conservation du patrimoine archéologique.

Par conséquent, en Espagne, l'intervention de l'administration est décisive dans tout ce qui affecte la recherche, la conservation, la tutelle et la valorisation du patrimoine archéologique en lien avec son caractère de domaine public, émanant d'un mandat constitutionnel et d'une législation qui souligne le caractère social de tout le patrimoine historique. Tout cela fait partie de la volonté de construire un «État de Bien-être» et représente une époque (fin des années 1970-début des années 1980) caractérisée par la transition démocratique et le consensus politique et social de tous les secteurs de la société espagnole. Toutefois, il est frappant de voir l'articulation d'un modèle d'archéologie préventive, dérivé du mandat du corpus législatif, et l'apparition de politiques néolibérales contenant des mesures de privatisation et affectant ainsi son organisation. Ce modèle a été promu par les départements du patrimoine d'une série de communautés autonomes, ce qui s'explique, en partie, par leur faible dotation en ressources humaines et leur incapacité à faire face aux responsabilités croissantes dont ils héritaient en matière de prévention et d'application de la législation, comme nous l'analyserons ci-après.

\section{Origines et phases du modèle d'archéologie}

préventive. Le développement économique espagnol entre la fin des années 1980 et la décennie passée, a supposé une vaste politique de rénovation et d'expansion urbaine, ainsi que la réalisation de grandes infrastructures en contextes urbain et rural. Les nécessités dérivées du plan d'aménagement urbain et des infrastructures ont produit l'extension du domaine d'activité archéologique, avec l'apparition de ce qui, en Espagne, a été connu sous les termes d' «archéologie de gestion ». Cela a produit, comme le mentionnait la loi, le développement obligatoire d'excavations archéologiques, en préalable aux constructions, avec l'assurance de leur financement. Les fouilles, qui ressortaient jusqu'alors au domaine public, tombent à ce moment-là entre les mains des promoteurs publics ou privés, ces-derniers étant majoritaires.

En Espagne, par conséquent, on est passé, à la fin des années 1980, du modèle d'intervention publique, qui avait défini la gestion et la recherche archéologique, à la privatisation croissante de ce modèle d'intervention préventive entrainant l'apparition de l' « Archéologie Commerciale». Plusieurs facteurs aident à comprendre la nature de ce changement à cette époque : l'incapacité, déjà citée plus haut, des nouveaux départements du patrimoine des administrations autonomes et municipales, étant donné leurs faibles ressources humaines et matérielles, pour gérer un volume d'interventions archéologiques toujours croissant; l'éclosion immobilière et de nouvelles planifications en matière d'urbanisme et d'infrastructures.

Face à ce manque de ressources pour accomplir les dispositions définies par les diverses législations sur la protection et le contrôle du patrimoine archéologique, ces départements ont choisi d'externaliser leur politique de prévention. Ils ont ainsi transmis le modèle d'intervention archéologique préventive aux entreprises privées d'archéologie qui commençaient alors à faire leur apparition, poussées en principe par l'administration. À partir de ce moment, le rôle de l'administration s'est vu réduit et recentré sur une démarche purement administrative, de contrôle et de prise de décision sur l'activité archéologique effectuée. En définitive, a été assumé le modèle néolibéral anglo-saxon qui donne la priorité à la privatisation du service (Parga Dans, 2011, p. 126), se basant pour leur part sur les expériences des États-Unis durant les années 1980 et de l'Angleterre au début des années 1990, expériences supposées à l'origine de l'«Archéologie Commerciale» appelée à tord, à ses origines, «Archéologie de Gestion ». Ces administrations espagnoles, en dépit des différentes optiques autonomes et d'une tradition de gestion et de conception publique du patrimoine en harmonie avec d'autres modèles publics (France, Italie), ont finalement choisi de promouvoir ces mesures de privatisation et de donner naissance à cette «Archéologie Commerciale». Par conséquent, la naissance de ce nouveau modèle a introduit l'apparition d'une nouvelle niche économique liée à l'exploitation des ressources archéologiques. L'archéologie commerciale, siège de l'initiative privée, gère aujourd'hui la majorité des interventions archéologiques préventives effectuées en Espagne.

Les stratégies préventives, dans l'archéologie espagnole, ont connues deux phases. La première d'entre elles, de 1985 à 2000, est celle qui a vu la réalisation d'une archéologie de sauvetage, centrée sur l'excavation de gisements localisés avant même d'effectuer les aménagements. La deuxième, avec un profil plus préventif, de 2000 à aujourd'hui, est caractérisée par la localisation préalable des gisements et leur inclusion dans le plan d'aménagement urbain et territorial (Querol, 2010, p. 209). Dans cette deuxième phase, la réalisation d'une intervention archéologique préalable est obligatoire et ses résultats seront déterminants dans l'avis de l'administration sur l'approbation ou le rejet d'un projet d'aménagement. Au moment de développer ses propres stratégies de prévention, le pays a analysé les autres expériences européennes, en Angleterre, en France et en Italie, depuis leurs débuts, ainsi que les différentes réglementations et cartes internationales. En Espagne, le caractère diagnostic de la dénommée «archéologie préventive» est mis en relation avec la planification urbanistique et des infrastructures dans les contextes urbains et ruraux, planification qui est aussi de la compétence des communautés autonomes. Ceci, corrélé 
à la diversité des situations normatives et réglementaires développées par ces communautés en matière d'archéologie, a rendu impossible la génération d'un modèle de gestion complet, pour l'application des stratégies préventives.

\section{Au cour du modèle : organisation} administrative, financement et entreprise. L'archéologie préventive espagnole s'est articulée autour de l'élaboration de cartes archéologiques, comme outil de localisation et d'identification chronologique des divers gisements, mais aussi comme premier instrument de prévention en rapport avec la planification urbaine. La plupart des législations autonomes ont élaboré des catégories de protection et d'identification qui permettent une première phase de diagnostic du registre archéologique.

La mise en œuvre d'une intervention est semblable pour les secteurs urbains et ruraux présentant un potentiel archéologique, avec la réalisation d'un programme d'inspection qui débute avec une phase d'évaluation du potentiel des restes archéologiques. Cette phase de diagnostic se pratique sur une partie réduite du sous-sol (rarement supérieure à $5 \%$ ) où se trouvent les indices d'un gisement archéologique. À cet effet, on effectue des sondages stratigraphiques avec l'emploi de machines lourdes, pour la plupart. Ce modus operandi a atteint un tel degré d'intensité, qu'il a été partiellement inclus dans certaines législations autonomes, spécialement dans les diverses normes juridiques régionales développées dans la seconde moitié de la décennie passée.

Postérieurement à cette phase initiale, l'administration autonome décide des possibles alternatives d'intervention : établissement d'aires de protection, intervention extensive ou élimination du registre archéologique. Dans le cas le plus fréquemment conseillé par l'administration, celui de l'élimination des restes archéologiques, le remaniement du sous-sol ou la destruction du registre archéologique est généralement accompagné de l'obligation de contrôle ou de suivi des travaux mécaniques, afin de confirmer la nonexistence de nouveaux restes archéologiques. Dans le cas, minoritaire, on l'on décide de la conservation du gisement, on opte pour une combinaison des différentes alternatives, par la protection ou l'établissement de secteurs de réserve et l'excavation partielle du reste du gisement identifié. Dans certains cas, un élargissement de l'excavation est réalisé postérieurement. En ce qui concerne des espaces archéologiques significatifs, pour lesquels on a décidé une conservation, ont aussi été développés des plans de mise en valeur et de valorisation semblables à ceux exécutés dans des gisements singuliers non soumis à la pression urbaine.

À partir de cette procédure administrative initiale se produit une division claire, spécialement visible dans le cadre urbain de l'activité archéologique et urbanistique. Après l'intervention, et si l'avis de l'administration est favorable à l'exécution du plan d'aménagement urbain, on accorde au promoteur une «Licence d'Euvre». Dans le même temps, la documentation du registre archéologique doit être concrétisée par une étude plus large de l'intervention, sans qu'il existe à ce jour un critère unifié définissant précisément quels sont les éléments fondamentaux que doit contenir cette étude. Il n'existe pas une analogie temporelle entre la libération de la ressource économique (le sol) et l'étude archéologique, la première restant automatiquement entre les mains du promoteur alors que l'étude ou la monographie archéologique peut être élaborée et achevée dans un délai pouvant aller jusqu'à 5 ans. Par conséquent, il n'y a pas de réversibilité matérielle de l'activité, dans la mesure où le gisement archéologique se positionne administrativement sur deux plans différents. Mais comment sont choisis les critères d'évaluation qui décident si un gisement doit donner lieu à une intervention, être protégé ou détruit? Dans la plupart des cas, cette décision revient principalement aux services administratifs et fait suite à une somme de critères peu explicites. Ces derniers se basent sur le contrôle exercé par les techniciens de l'administration dans le cadre de leurs visites de chantier et, fondamentalement, sur les divers rapports produits par les archéologues engagés par le promoteur et qui, dans la plupart des cas, sont déterminants dans la décision de conservation ou de destruction des gisements archéologiques.

Le financement vient pour l'essentiel de promoteurs privés, ceux provenant du secteur public étant généralement minoritaires. Ce financement est obligatoire et il est imposé par les administrations aux promoteurs qui prennent en charge les frais d'intervention. Toutefois, les administrations n'en fixent pas le prix, donnant lieu à un marché non régulé où le budget est fixé à partir de la relation contractuelle qui est décidée entre le promoteur et les entreprises d'archéologie. Cette relation entre le financement de l'activité archéologique et les acteurs directement «touchés» par celui-ci, est l'un des problèmes pour lequel aucune solution satisfaisante n'a été trouvée et qui affecte clairement les résultats des interventions. Â ce propos, il faut signaler que, dans ce modèle de financement, le paiement de la recherche n'est pas prévu, ce qui explique que la majorité des interventions effectuées n'aient pas donné lieu à des travaux de recherche et n'ont pas non plus fait l'objet de monographie. En découle que la majorité de la documentation existante sur les gisements archéologiques est limitée à la rédaction d'un rapport obligatoire, sans que puisse être envisagée une étude en profondeur ni une alternative à la monographie scientifique, dont la rédaction est pourtant aussi envisagée par la réglementation. Toutefois, il a déjà été mis en évidence qu'on ne peut tenir pour responsables de ce fait les archéologues engagés sur ces opérations, puisque cette limitation leur est imposée par un système qui n'oblige pas à payer la phase de recherche et, par conséquent, le fait de l'effectuer les condamnerait à de longues périodes d' «inactivité» qui leur sont impossibles (Salvatierra, Sous presse).

Limpact direct en matière de diffusion des résultats des interventions constitue un problème sérieux et de grande portée. L'administration, de fait, oblige à la réalisation d'une monographie mais, sauf exception, elle ne met pas les moyens normatifs suffisants pour la mener à bien. À ceci, il faut ajouter 
l'absence de mécanismes qui obligeraient à inclure, dans le budget général, des volets pour la recherche. Toutefois, quelques communautés autonomes et mairies publient dans leurs séries périodiques une sélection d'interventions préventives, ainsi qu'un nombre réduit de monographies concernant les activités les plus significatives. Il y a aussi des entreprises qui font l'effort de publier une sélection de leurs interventions sous forme d'articles ou, dans une moindre mesure, d'ouvrages. Certaines d'entre elles introduisent le résultat de leurs travaux dans le cadre d'études universitaires ou d'organismes publics de recherche. Malgré ces initiatives, on souligne tout de même une nette pénurie d'études en proportion de l'énorme volume d'activités réalisées durant les deux dernières décennies.

La constitution d'entreprises a aussi impliqué un traitement différencié du travail professionnel à effectuer, avec différents degrés de compromis face au patrimoine archéologique et un certain relâchement des mécanismes de contrôle par l'administration responsable de l'archéologie dans une grande partie des communautés autonomes. Tout cela est la conséquence du modèle appliqué qui a donné la priorité à la construction au détriment de la conservation du patrimoine archéologique ; modèle dans lequel l'administration exerce seulement les tâches d'autorisation, de contrôle et de décision. Dans tout ce processus, il faut signaler qu'il n'y a pas de régulation des relations contractuelles entre les promoteurs et les entreprises d'archéologie, celles-ci se développant dans un cadre non régulé; comme quoi l'expression «celui qui détruit paie» devrait être complétée par «celui qui détruit paie et impose». De fait, cela produit des situations où les promoteurs n'embauchent pas les entreprises qui fouillent le mieux, mais celles qui présentent le projet le moins cher, ce facteur étant généralement inversement proportionnel à la qualité de l'intervention (Salvatierra, Sous presse).

Les entreprises d'archéologie en Espagne s'intègrent dans le secteur de la petite entreprise. Selon les données d'une étude récente sur l'archéologie commerciale (Parga Dans, 2010, p. 206), réalisée en 2008, ces entreprises employaient un total de 2358 personnes : 457 des chefs d'entreprise, 573 travailleurs fixes et 1328 temporaires (sans compter les travailleurs autonomes), avec une prédominance des contrats à durée déterminée. L'enquête a été effectuée sur un total de 273 entreprises (dont 212 ont répondu à l'appel), chiffre correspondant à ce qui était estimé en Espagne durant cette année; à noter que ce chiffre a diminué depuis, notamment suite à la crise. Les communautés autonomes présentant le plus grand nombre d'entreprises étaient l'Andalousie, la Catalogne et la région de Madrid, et les villes de plus grande densité telle que Madrid et Barcelone. En moyenne ces entreprises sont constituées d'un (25\%) ou deux (47\%) chefs d'entreprise, d'un nombre réduit de technicienarchéologues en contrat à durée indéterminée ( 2 employés en moyenne) et d'une majorité de travailleurs contractuels engagés en fonction des projets en cours dans l'entreprise (Parga Dans, 2010, p. 191-193 et p. 201-206). Cette structure, qui a généré une forte précarisation du travail et de la masse salariale, définit le cadre des relations contractuelles à laquelle sont soumis les employés des entreprises. À titre d'exemple, des conventions collectives ont seulement été signées dans 3 des 17 communautés autonomes : la Catalogne (2007), la Galice (2009) et Castille-et-León (2010). Tout cela est la conséquence d'un marché basé sur la libéralisation des prix, ce qui suppose une réduction des budgets et affecte de manière déterminante les travaux de diagnostic, d'exécution et d'étude des restes exhumés.

Il faut mentionner que, grâce à l'expansion de l' «Archéologie Commerciale », l'archéologie a connu en Espagne un développement spectaculaire durant ces dernières années. Toutefois, son principal poids en termes de nombre d'interventions a été gonflé par une conjoncture d'opportunités, généralement étrangères à une planification rationnelle répondant aux nécessités et objectifs de ces interventions ou à des programmes de recherche concrets. Les interventions archéologiques de ce type se sont transformées en une pièce convoitée dans un marché réellement incontrôlé. Malheureusement, dans la majorité des cas, ce type d'intervention reste une simple justification administrative sans finalité de recherche et d'enrichissement de la connaissance historique, pourtant inscrite dans le mandat constitutionnel (art. 46) et la législation dérivée sur le patrimoine historique ; l'archéologie s'est ainsi vue pliée aux exigences et à la pression du marché immobilier, en se transformant, pratiquement, en une simple démarche administrative. (AAVV, 2000, p. 69). L'autre grave problème qui en découle est le fait que l'archéologie espagnole fait aujourd'hui face à une dissociation entre la recherche archéologique et l'archéologie commerciale.

Avec ce modèle, l' «Archéologie Commerciale» voit son domaine d'activité relié aux aménagements urbains et de grandes infrastructures, en ayant pour priorité le sauvetage ou la documentation, pour son élimination postérieure, des restes archéologiques, tout en ne devant pas répondre à une méthodologie complexe ni employer de techniques sophistiquées de recherche, si celles-ci empêchaient une extraction rapide des restes. Au contraire, l' «Archéologie de Recherche», attachée au savoir académique, est en charge de développer des méthodes interdisciplinaires complexes et des campagnes d'excavation plus lentes, non soumises à la pression d'un possible conflit avec l'aménageur, son activité étant plus généralement développée en contexte rural, avec pour objectif des connaissances historiques (AAVV, 2000, p. 66). On en est ainsi arrivé à la situation actuelle, où les archéologues de l'université et des organismes de recherche, d'une part, et une grande partie des archéologues de l' «Archéologie Commerciale», d'autre part, constituent deux groupes de personnes et d'intérêts distincts (Salvatierra, Sous presse).

Ce conflit entre l'Archéologie Académique, ou de recherche, et l'Archéologie Commerciale, qui n'est pas seulement caractéristique de la réalité 
espagnole, puisqu'il a aussi été généré aux États-Unis et au Royaume-Uni, est en réalité un conflit faux et intéressé. Le divorce entre ces deux «archéologies » est fondé sur l'hypothèse de base voulant que ses professionnels aient des objectifs différents dans le traitement de la pratique archéologique, dans un cadre archéologique propice aux politiques néolibérales. Déjà en son temps, nous interrogions un vaste groupe de spécialistes dans un volume collectif sur l'administration et le bénéfice du patrimoine historique espagnol (AAVV, 200o, p. 65-72; Orme, Enciso, 2002, p. 245). Cette critique était fondée sur la défense et la considération que tout ce qui alimente notre connaissance historique provient du travail d'investigation. On fait des recherches non pas seulement pour connaître le passé, mais aussi pour le restaurer, le conserver, le diffuser et le présenter de manière didactique, en favorisant, par conséquent, le bénéfice de la société sur son patrimoine historique.

Par conséquent, tous ces comportements, indépendamment des critères (intérêts scientifiques, rentabilité sociale...) ou de la conjoncture (risque, planification...), qui ont primés en vue de la décision finale de réalisation, de non-réalisation ou de recherche, ne relèvent pas d'une démarche archéologique. Il faut défendre le fait que la gestion archéologique doit comprendre, favoriser et développer ce caractère investigateur. Pour cela il faut supprimer ces dichotomies provoquées par la privatisation des interventions archéologiques, comme celle existante entre l'Archéologie de Recherche et l'Archéologie Commerciale, et favoriser la vision unique d'une archéologie comme vecteur de recherche à tous ses niveaux (AAVV, 2000, p. 70).

Tout ce qui a été dit jusqu'ici reflète une réalité complexe. D'une part, il est nécessaire de souligner comment, durant les vingt dernières années, la situation du patrimoine archéologique a positivement changé, même si l'on est encore loin d'atteindre le niveau souhaitable. Il peut être fait valoir que, dans l'ensemble, dans une partie appréciable des villes espagnoles possédant un important patrimoine archéologique, on a progressé de manière remarquable en matière de recherche, de tutelle, de conservation et d'intégration des restes archéologiques et, par conséquent, dans la connaissance de l'évolution historique. Mais, malgré ces avancées, il est également certain que l'on a assisté à une sensible perte de patrimoine suite à l'expansion et à la rénovation urbaine, ainsi qu'à une importante politique de construction d'infrastructures sur le territoire. Tout en récapitulant la situation de l'archéologie préventive en Espagne, à partir d'une vision générale, il faut souligner la diversité des circonstances existantes, conséquences de plusieurs facteurs : - les différents degrés d'application et d'exécution; - la réglementation légale appliquée par les différentes administrations (nationale, autonome, locale); -le manque d'une politique, par ces mêmes administrations, basée sur des projets de gestion homogènes au moment de poser les stratégies de prévention, de recherche, de conservation ou d'élimination, et de valorisation du patrimoine archéologique urbain;
- le manque de coordination entre les différentes administrations en vue d'une gestion correcte du patrimoine archéologique;

- une étonnante pénurie en matière de plans de recherche et de formation en archéologie préventive par les différentes institutions scientifiques;

- l'absence d'intervention de l'administration comme garant des conditions minimales en matière de financement des projets, ainsi que des conditions de travail des archéologues.

En ce sens, il convient de remarquer le consensus généralisé entre les spécialistes favorables à une standardisation d'un point de vue légal et méthodologique, qui développe des directives de base homogènes, consensus devant émaner de l'environnement administratif lui-même (Parga Dans, 2010, p. 183).

Tout cela pourrait faire l'objet d'un débat, basé sur les observations de ces dernières années, sur le futur de ce qu'on a appelé «archéologie préventive» en Espagne; on y défendrait la nécessité d'influencer et d'étendre ledit caractère préventif (AA.VV, 2000; Salvatierra, 2004 et Sous presse; Olmo Enciso, 2002; Quirós, 2005). Ceci suppose de parier sur un projet interdisciplinaire qui, promu par les administrations, miserait sur la coordination et le travail conjoint entre les universités, les organismes publics de recherche, les associations de travailleurs de l'archéologie et les administrations compétentes. Peut-être l'un des aspects les plus intéressants serait-il de faire prendre part l'archéologie aux processus conceptuels, projectifs et normatifs qui orientent la transformation du milieu urbain et territorial ? Cela suppose la revendication de l'archéologie comme une activité scientifique très actuelle et, par conséquent, partie prenante du débat sur la configuration de la société d'aujourd'hui. Il faut dépasser le cadre réducteur d'une archéologie soumise aux directives marquées par la planification urbaine et territoriale, ainsi que par les nécessités du «marché», car c'est malheureusement ce dernier qui a caractérisé jusquà présent l'«archéologie préventive» espagnole. La solution ne se trouve pas dans la réprobation des conséquences du modèle, mais dans la critique des origines de celui-ci. Il ne faut pas se concentrer sur les instruments, mais sur les objectifs qui les ont générés.

AAVV, 2000 : Un futuro para la memoria. Sobre la administración y disfrute del Patrimonio Histórico Español, Madrid, éd. Visor.

GARCía FernÁNDEZ J., 2006 : Estudios sobre el Derecho del Patrimonio Histórico, Madrid.

Olmo ENCISO L., 2002 : «Arqueología y modelos de ciudad: una reflexión desde España», in RICCI A. (ÉD.), Archeologia e Urbanistica, Firenze, éd. All'Insegna Del Giglio, p. 243-256.

PARga DANS E., 2010 : Innovación y emergencia de un servicio intensivo en conocimiento: el caso de la Arqueología Comercial. Santiago de Compostela, Universidad de Santiago de Compostela.

Querol M.A., 2010 : Manual de Gestión del Patrimonio Cultural, Madrid, éd. Akal.

Querol M.A., MARTínez DíAz B., 1996 : La gestión del Patrimonio Arqueológico en España, Madrid, éd. Alianza.

Quirós CASTILLO J.A., 2005: «¿Excavar en las ciudades o historiar las ciudades? El debate sobre la Arqueología Urbana a la luz de algunas experiencias europeas ", Arqueología y Territorio Medieval, n ${ }^{12.1}$, Jaén, p. 107-130.

SALVATIERRA V., 2004: «Arqueología urbana. Investigación y profesión », Anales de Arqueología Cordobesa, vol. 15, Córdoba, p. 45-59.

SAlVATIERRA V., Sous presse : «Arqueología y Urbanismo », in AAVV, La Materialidad de la Historia, Madrid, éd. Akal. 